\title{
BEAM POSITION MONITOR OFFSET DETERMINATION AT LEP
}

\author{
F. Tecker, RWTH Aachen, Germany, \\ B. Dehning, P. Galbraith, K. Henrichsen, M. Placidi, R. Schmidt, CERN, Geneva, Switzerland
}

\begin{abstract}
For the performance of an electron positron storage ring it is important that the beam orbit passes well centred through the quadrupole magnets. Beam position monitors (BPM) aligned relative to the magnets can still have a residual mechanical or electronical offset with respect to the magnetic axis. A beam-based method is used at LEP to measure these offsets. During the machine operation for physics the gradient of selected quadrupoles is modulated with low frequencies (few $\mathrm{Hz}$ ) and very small amplitudes (of the order of $10^{-4}$ ). The effect on the beam is observed with a high sensitivity pick-up. The observed effect passes through a minimum when the beam is centred in the quadrupole. Offsets for about 70 different BPM's were determined. Systematically different offsets were found for different type of BPM electronics and different types of quadrupoles. Simulations based on the past results show that the level of spin polarisation can be increased by further offset measurements.
\end{abstract}

\section{INTRODUCTION}

An important issue for the successful operation of a particle accelerator is an efficient control of the closed orbit. Luminosity, background in the physics experiments and the degree of beam polarisation depend strongly on the vertical closed orbit. The best performance is achieved if the quadrupoles are well aligned and the beam travels through the magnetic centre of the quadrupoles.

The quadrupole magnets form a reference system for the beam and are aligned with high precision. Beam position monitors (BPM) at the quadrupoles can have a mechanical or electronical offset with respect to the magnetic centre of the magnets. This offset is measured by a beam based method [1].

\section{PRINCIPLE OF BEAM BASED ALIGNMENT}

The principle of the methods for beam based alignment is based on the fact that the magnetic field inside a quadrupole magnet increases linearly with the distance from the centre. When the gradient is varied, the field change is proportional to the distance from the magnetic axis of the quadrupole. An off-axis beam travelling through the magnet receives an additional deflection depending on its position. The gradient changes are slow compared to the betatron frequency. The closed orbit variation can be measured at nearly any location in the ring. This effect vanishes when the beam passes centred through the quadrupole. A more detailed description of the method used at LEP can be found in [2].

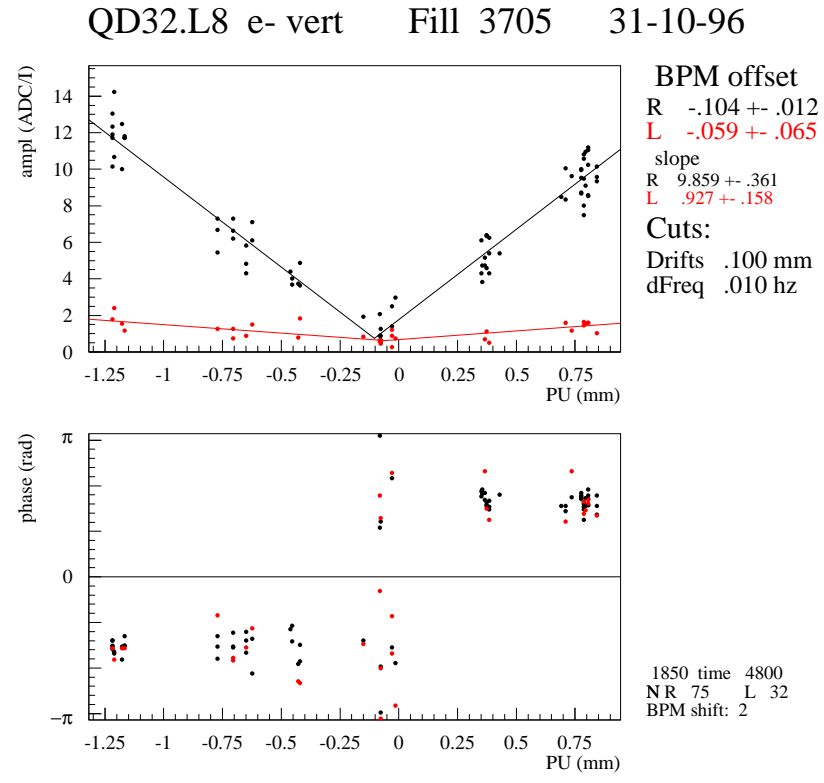

Figure 1: Oscillation amplitude (top) and phase (bottom) measured by the two couplers as a function of the beam position monitor reading

The strength of the quadrupole magnet is modulated periodically at frequencies in the range from 0.7 to $16 \mathrm{~Hz}$. Since most of the magnets are powered in series, additional (back leg) winding have been installed to change the gradient of individual quadrupoles. The quadrupoles in all straight sections and in one of the eight LEP arcs were equipped with back leg windings. The modulation depth is smaller than $10^{-3}$ and the measurements can be performed in parallel to the physics experiments' data taking. The offsets of several BPMs can be measured simultaneously using different excitation frequencies.

The induced beam oscillations are measured by two sensitive beam position monitors (couplers) close to an odd multiple of $\pi / 2$ phase advance apart to be able to detect every oscillation with acceptable sensitivity. A harmonic analysis of the signal determines the oscillation amplitude at the excitation frequency. This amplitude is proportional to the distance from the axis of the quadrupole with the modulation and shows a V-shaped behaviour when plotted as function of the beam position in the quadrupole. The position changes due to orbit drifts [3] or by intentional amplitude variations of local closed orbit bumps. Fig. 1 shows the results of an offset determination by the two couplers. The measurement points show some scatter since 
beam oscillations from other sources than the modulation are always present. Different cuts are applied to improve the data quality. A three parameter fit gives the offset of the BPM. The amplitude for one of the couplers is smaller due to a less favourable betatron phase advance between magnet and coupler, and the results are less precise. Since the modulation of the magnet current and the measurement are synchronised, one can determine the phase of the orbit oscillation with respect to the modulation. The measured phase changes by $\pi$ when crossing the symmetry plane of the quadrupole which gives an additional crosscheck.

\section{DISTRIBUTION OF OFFSETS}

The beam position monitor system of LEP uses two different types of electronics, a narrow band and a wide band system. The BPMs close to the interaction points have the wide band electronic to cope with the shorter time separation between the counterrotating bunches. The wide band BPM have different gain settings depending on the beam current. All other BPMs, in particular the BPMs in the arcs of LEP, have narrow band electronics which is independent of the beam current.

Eight quadrupoles (QS0) at the four interaction regions of LEP are superconducting magnets to achieve the strong gradient needed for the focusing at the interaction points. The associated BPMs are of the wide band type. Their offsets were found to be significantly larger than for any other BPMs. The first generation of superconducting quadrupoles at LEP had systematic offsets up to nearly $2 \mathrm{~mm}$. After replacing these magnets with the new generation of quadrupoles for LEP2, the measured offsets were below $1 \mathrm{~mm}$. The wide band BPMs show different offsets for different gain settings and for the two particle types. Differences of up to $300 \mu \mathrm{m}$ were measured. This implies that the offset is partially caused by the BPM processing electronics.

The wide band BPMs at normal conducting magnets show smaller offsets than those at the superconducting quadrupoles. A calibration was performed for a large number of arc quadrupoles with narrow band electronics BPMs. In general, the offsets are smaller than for the wide band electronic and the differences between the two particle types are less important. Table 1 compares the offsets for the different categories. The measured offsets are included as a correction to the BPM readings whenever an orbit is acquired.

\begin{tabular}{|l|c|c|c|}
\hline \multicolumn{2}{|c|}{} & RMS $[\mu \mathrm{m}]$ & mean $[\mu \mathrm{m}]$ \\
\hline \hline \multirow{2}{*}{ wide band } & QS0 & 250 & -600 \\
\cline { 2 - 4 } & other & 350 & -220 \\
\hline \multicolumn{2}{|c|}{ narrow band } & 185 & -90 \\
\hline
\end{tabular}

Table 1: Characteristics of offset distribution for different magnet types and BPM electronics



Figure 2: Relevant offsets for the BPM misalignment relative to the reference plane

\section{IMPACT ON POLARISATION LEVEL}

The vertical orbit is of particular importance for a high level of beam polarisation. The spins of the particles in an electron-positron storage ring tend to polarise in the vertical direction with time. Horizontal fields from vertical corrector dipoles and a beam passing off-centre in the quadrupoles can create depolarising resonances. The depolarising effects become significantly stronger at higher beam energies [4].

In order to maximise the degree of polarisation, the resonances can be compensated by so-called Harmonic Spin Matching (HSM) [5]. Two different variations of HSM exist:

deterministic HSM: A Fourier analysis of the vertical closed orbit $[6,7]$ gives the strength of the necessary orbit bumps (4 parameters).

empirical HSM: The 4 parameters of the bump amplitudes are varied and the changes in the polarisation level are observed.

In principle, empirical HSM could be used to find the best compensation for the depolarising resonances. In practical application it is very tedious and time consuming since one has to find the optimum in a four-dimensional parameter space and the build-up time for the polarisation is slow. If the initial polarisation level is too low, it is even impossible to observe changes of the polarisation level when the parameters are varied.

For this reason the deterministic HSM has to be applied. The precision of the calculated parameters depends strongly on the BPM offsets relative to a reference plane. Simulations with the SITF [8] module of MAD [9] were performed to study the effects in detail. In the simulations, the quadrupoles were misaligned with an RMS value of $\sigma\left(\Delta y_{\text {quad }}\right)=150 \mu \mathrm{m}$ which corresponds to the value measured by the geometrical survey. The RMS values of the BPM offsets $\sigma\left(\Delta y_{k-m o d}\right)$ to the quadrupole axis was varied between 0 and $700 \mu \mathrm{m}$. The different offsets are illustrated in Fig. 2. The offset of the BPM relative to the reference orbit in a plane is

$$
\Delta y_{B P M}=\Delta y_{q u a d}+\Delta y_{k-\text { mod }}
$$

Ten different random distributions were used for each RMS value of the offsets. The orbit was corrected to the best 




Figure 3: Influence of the vertical offset between BPM and quadrupole axis on the polarisation level.

possible value. The harmonic content of the vertical BPM readings were analysed. The polarisation level in linear approximation was determined by SITF before and after applying HSM.

The linear calculations of SITF do not give a realistic absolute value of the polarisation level. Higher orders can be included [4]. This was done for a few samples. Therefore the average value of the ten random seeds for the linear results are normalised to the average level of polarisation obtained by empirical HSM. This is the highest level of polarisation achievable for a given energy and set of tunes. The polarisation level for empirical HSM was calculated in the simulations by deterministic HSM taking the beam position with respect to the reference orbit without errors. The results for the beam energy of $55 \mathrm{GeV}$ are shown in Fig. 3.

The polarisation level without HSM is only slightly dependent on the BPM offsets. The polarisation level with deterministic HSM increases with decreasing BPM offset but saturates at about 0.55 for $\sigma\left(\Delta y_{k-\text { mod }}\right)<150 \mu \mathrm{m}$. This can be understood since then the error of the position of the BPM relative to the reference plane $\sigma\left(\triangle y_{B P M}\right)$ is dominated by the misalignment of the magnets $\sigma\left(\Delta y_{\text {quad }}\right)$ since

$$
\sigma\left(\Delta y_{B P M}\right)=\sqrt{\sigma\left(\Delta y_{\text {quad }}\right)^{2}+\sigma\left(\Delta y_{k-\text { mod }}\right)^{2}} .
$$

The vertical mechanical misalignment of the quadrupole is measured with a precision of about $50 \mu \mathrm{m}$ at LEP. This information can be used to get a more precise knowledge of the BPM relative to the reference plane. The achievable error of the offset determination by the beam based alignment has the same magnitude. The deterministic HSM including the geometrical alignment data was simulated (see again Fig. 3).

The polarisation level does not show the saturation effect and 0.8 of the level by empirical HSM can be obtained with $\sigma\left(\Delta y_{k-\text { mod }}\right)=50 \mu \mathrm{m}$. This value can be achieved if all BPM offsets in the arcs of LEP are measured. The gain in polarisation will be only minor when the mechanical measurements are included without the offset determination since then the error $\sigma\left(\Delta y_{B P M}\right)$ is dominated by the BPM offsets.

\section{CONCLUSIONS AND OUTLOOK}

The Harmonic Spin Matching technique becomes more precise by measuring the offsets of all BPMs in the arcs of LEP and including the measured misalignment of the quadrupole magnets at LEP from the geometrical survey. Simulations show that the optimum level of polarisation for a given energy can nearly be reached by deterministic HSM. The remaining optimisation can be performed by empirical HSM.

Presently, the all quadrupoles at LEP with an associated BPM are being equipped with back leg windings. It is envisaged to measure the offsets of all BPMs during the 1997 running period.

\section{REFERENCES}

[1] D. Rice et al., Beam Diagnostic Instrumentation at CESR, IEEE Transitions on Nuclear Science, NS-30(4):2190, 1983.

[2] I. Barnett, A. Beuret, B. Dehning, P. Galbraith, K. Henrichsen, M. Jonker, M. Placidi, I. Reichel, R. Schmidt, F. Tecker, L. Vos, and J. Wenninger, Dynamic Beam Based Calibration of Orbit Monitors at LEP, In Proceedings of the Fourth International Workshop on Accelerator Alignment, KEK Proceedings 95-12, pages 421-426, 1995, CERN SL/95-97 (BI).

[3] W. Coosemans, A. Marin, K. Rybaltchenko, F. Tecker, and J. Wenninger, Closed Orbit Feed-Back from low-beta Quadrupole Movements at LEP, this Conference.

[4] A. Blondel, M. Böge, B. Dehning, A. Drees, M. Placidi, F. Tecker, and J. Wenninger, Transverse Polarization beyond the Z Energy at LEP, this Conference.

[5] R. Rossmanith and R. Schmidt, Compensation of Depolarizing Effects in Electron Positron Storage Rings, Nucl. Instr. Meth. Phys. Res., A(236):231, 1985.

[6] R. Assmann, Optimierung der transversalen Spin-Polarisation im LEP-Speicherring und Anwendung für Präzisionsmessungen am Z-Boson, $\mathrm{PhD}$ thesis, Ludwig-MaximiliansUniversität München, 1994.

[7] R. Assmann et al., Polarization Studies at LEP in 1993, CERN SL/94-08 (AP).

[8] J. Kewisch et al., Phys. Rev. Lett., 62:419, 1989.

[9] H. Grote and F. Iselin, The MAD program, CERN SL/90-13 (AP). 